\title{
Erratum to: Handwriting Difficulties in Children with Autism Spectrum Disorders: A Scoping Review
}

\author{
Azadeh Kushki - Tom Chau - Evdokia Anagnostou
}

Published online: 24 July 2011

(C) Springer Science+Business Media, LLC 2011

\section{Erratum to: J Autism Dev Disord}

\section{DOI 10.1007/s10803-011-1206-0}

Please note that the Cartmill et al. (2009) reference was provided incorrectly in the published article. The correct reference is:
Cartmill, L. J., Rodger, S., \& Ziviani, J. (2009). Handwriting of eight year old children with autistic spectrum disorder: An exploration. Journal of Occupational Therapy, Schools and Early Intervention, 2, 103-118.

The online version of the original article can be found under doi:10.1007/s10803-011-1206-0.

\footnotetext{
A. Kushki · T. Chau · E. Anagnostou ( $\square)$

Bloorview Research Institute, Holland Bloorview Kids

Rehabilitation Hospital, 150 Kilgour Road, Toronto,

ON M4G 1R8, Canada

e-mail: eanagnostou@hollandbloorview.ca

A. Kushki

e-mail: azadeh.kushki@utoronto.ca

T. Chau

e-mail: tchau@hollandbloorview.ca
} 